\title{
Low cost double optical tweezers to manipulate microorganisms and micro-crystals
}

\section{Pinza óptica doble de bajo costo para manipular microorganismos y micro-cristales}

\author{
J. Vargas Valencia ${ }^{1,2 *}$, Jean Pierre Galaup ${ }^{3}$
}

1. Instituto Tecnológico Metropolitano de Medellín ITM, Sede Boston, Calle 50A N³0-01, Medellín, Colombia.

2. Instituto de Física, Universidad de Antioquia, Calle $67 N^{\circ}$ 53-108, Bloque 6, oficina 105. Medellín, Colombia. 3 Laboratoire Aimé Cotton, Université Paris-Sud 11, Centre National de la Recherche Scientifique CNRS, Campus d'Orsay-Bâtiment 505, 91405 Orsay Cedex, France.

${ }^{(*)}$ E-mail: javiervargas@itm.edu.co

Received: 24/11/2015 Accepted: 15/09/2016

DOI: 10.7149/OPA.49.4.48551

\begin{abstract}
:
An inexpensive double optical tweezers has been used for trapping and manipulating microorganisms and micro-sized crystals with regular and irregular shapes. The intensity and spatial location of the traps were controlled through mirror displacements devices. A stable infrared laser beam with tunable power was used. The particles were manipulated and observed in an inverted microscope adjusted to locate the infrared trapping laser beam into the holder. A bacilliform bacterium with an approximate size of $12.0 \mu \mathrm{m}$ was captured by its two elongated ends, exerting control of their displacements and rotations. Also, regular micro-crystals measuring approximately $10.0 \mu \mathrm{mx} 4.0 \mu \mathrm{mx} 0.5 \mu \mathrm{m}$ were captured, achieving nanometric control of their displacements and rotations in two dimensions, parallel to one of their faces.
\end{abstract}

Key words: optical tweezers, micromanipulation, micro crystals, micro machines.

\section{REFERENCES AND LINKS}

[1] A. Ashkin, "Acceleration and trapping particles by radiation pressure", Phys Rev Let 24, 156-159 (1970). https://doi.org/10.1103/PhysRevLett.24.156

[2] W. D. Phillips, "Laser cooling and trapping”, Reviews of Modern Physics 70, 721-741 (1998). https://doi.org/10.1103/RevModPhys.70.721

[3] M. J. Lang, S. M. Block, “Resource Letter: LBOT-1: Laser-based optical tweezers”, Am J Phys 71, 201215 (2003). https://doi.org/10.1119/1.1532323

[4] A. Ashkin, "History of Optical Trapping and Manipulation of Small-Neutral Particle, Atoms and Molecules”, IEEE Journal on Selected Topics in Quantum Electronics 6, 841-856 (2000). https://doi.org/10.1109/2944.902132

[5] S. E. Lyshevski, MEMS and NEMS: Systems, Devices and Structures, CRC Press LCC (2002).

[6] S. P. Smith, S. R. Bhalotra, A. L. Brody, B. L. Brown, E. K. Boyda, M. Prentiss, "Inexpensive optical tweezers for undergraduate laboratories", Am J Phys 67, 26-35 (1999). https://doi.org/10.1119/1.19187 
[7] M. Rodriguez Otazo. "Réalization des pinces optiques pour la manipulation de nano et micro objets individuels d'intérêt chimique ou biologique", Thèse pour obtenir le grade Docteur de l'Université Paris Sud 11, 109-112, Juillet 2008.

[8] D. McGloin. “Optical tweezers: 20 years on”, Phil Trans, R Soc A 364, 3521-3537 (2006).

\section{Introducction}

Optical tweezers are high precision devices which use radiation pressure to capture, manipulate, and exert controlled forces in nano and pico scales and to carry out a wide range of experiments that involve micro and nano particles besides microorganisms. These tweezers evolved significantly in their technical aspects and applications since 1970, when Ashkin published the first report of the use of a visible Argon laser to accelerate dielectric spheres on micrometric scale [1]. Ashkin's first reports also proposed the possible use of radiation pressure to carry out experiments with atoms and molecules. These experiments were later performed by Claude Cohen Tannoudji who shared the Nobel of Physics in 1997 with Steven Chu and William D. Phillips for the research of atoms cooling and Bose-Einstein condensates [2].

Currently, optical tweezers make possible a wide variety of trapping techniques and can be used in different application fields, allowing the manipulation of bodies with dimensions that can vary from sizes of atoms or molecules until orders of several hundreds of micrometers, including nanostructures, microstructures and microorganisms such as bacteria, cells and cells organs. More recently, they have been used for investigations in genetics and related fields $[3,4]$. They can also be important in the investigation of materials, chemistry, atomic physics, solid state physics, etc. [4]. The possibility of micro and nanomanipulation offered by tweezers opens the door to their use in the construction of microstructures and micro machines known as micro-electromechanical systems (MEMS) or simply microsystems (MS) [5]. Most of the publications in this area present results concerning the manipulation of spheres of micrometric diameter and about the characterization of several aspects in relation to the optical traps for these spheres. However, there are a few low-cost alternatives that permit the spatial control of the objects with other geometrical shapes, including regular and irregular crystals. In this sense, our work aims to provide a contribution consisting of a low-cost research alternative that allows to make investigations to scientific communities with low resources, similar to ours.

In this work the results of the two dimensional manipulation of microorganisms and dielectric microcrystals via optics are exhibited. Low cost double optical tweezers that use a power-modulated infrared (IR) laser, a dividing system to generate the two traps, a telescopic mechanism of focus, an inverted microscope with an 100X objective lens and a system of image capture, were constructed and used to achieve micromanipulation in two dimensions.

\section{Optical Trap Devices}

For our system an infrared monomode laser manufactured by IPG Photonics with a maximum power of 1 $\mathrm{W}$ was used. This laser $\mathrm{TEM}_{00}$ is linearly polarized and collimated with a diameter of about $6 \mathrm{~mm}$ and 1.06 $\mu m$ wave-length. In biological investigations IR lasers are commonly used because they are considered noninvasive, while producing the minimum or no damage in alive organisms at the moment of capture. On the other hand, microorganisms absorb visible radiation, incrementing their temperature and dying afterwards. The assembly of the double tweezers is shown in figure 1, where all its parts are illustrated.

The linearly polarized laser beam leaves the optic fiber from the part $A$ of the figure 1 . Then, it goes through a polarization rotor at $\mathrm{B}$ that will be used later to balance the power distribution in the traps if necessary, controlling the orientation of polarization $P$ (see fig. 2). Part C has two beam splitters (BS) and two mirrors. The first BS divides the laser beam into two components perpendicularly polarized which will be used to obtain the two individual traps inside the microscope (perpendicular polarizations $P_{1}$ and $P_{2}$ ). The two mirrors reflect each of these rays toward the second BS which is used as collector. In order to control the position of each trap inside the microscope, mechanical controls of mirror orientation were used.

Part D is a telescopic system that is commonly used to focus the laser beam at the microscope objective entrance [6]. Two plane-convex lenses with equal focal distance $f$ separated by a distance $2 f$ were used. The entrance of the objective is located at a distance $f$ from the second lens of the telescopic system. It was necessary to place a dichroic mirror in the lower part of the microscope which allows the reflection of the infrared laser through the objective entrance, but preventing its entrance in the image capture system to avoid any possible damage to the camera. It is also necessary to place a birrefringent crystal between the two lenses of the telescopic system D so that the dichroic mirror at the microscope reflects both components 
perpendicularly polarized of the incident ray to 45 grades, because the dividing system $C$ sends a component horizontally polarized and the other one vertically, and these may not be reflected with the same intensity in the dichroic mirror. Part E is an inverted microscope Nikon Ellipse 100 which has a lens with the following characteristics: NA=1.25, magnification objective $100 \mathrm{X}, \mathrm{WD} 0.23$, infinity/0.17 in whose holder the capture is carried out. Finally, part F is a CCD camera which should also have additional protection filters to protect the camera from high power laser beams.

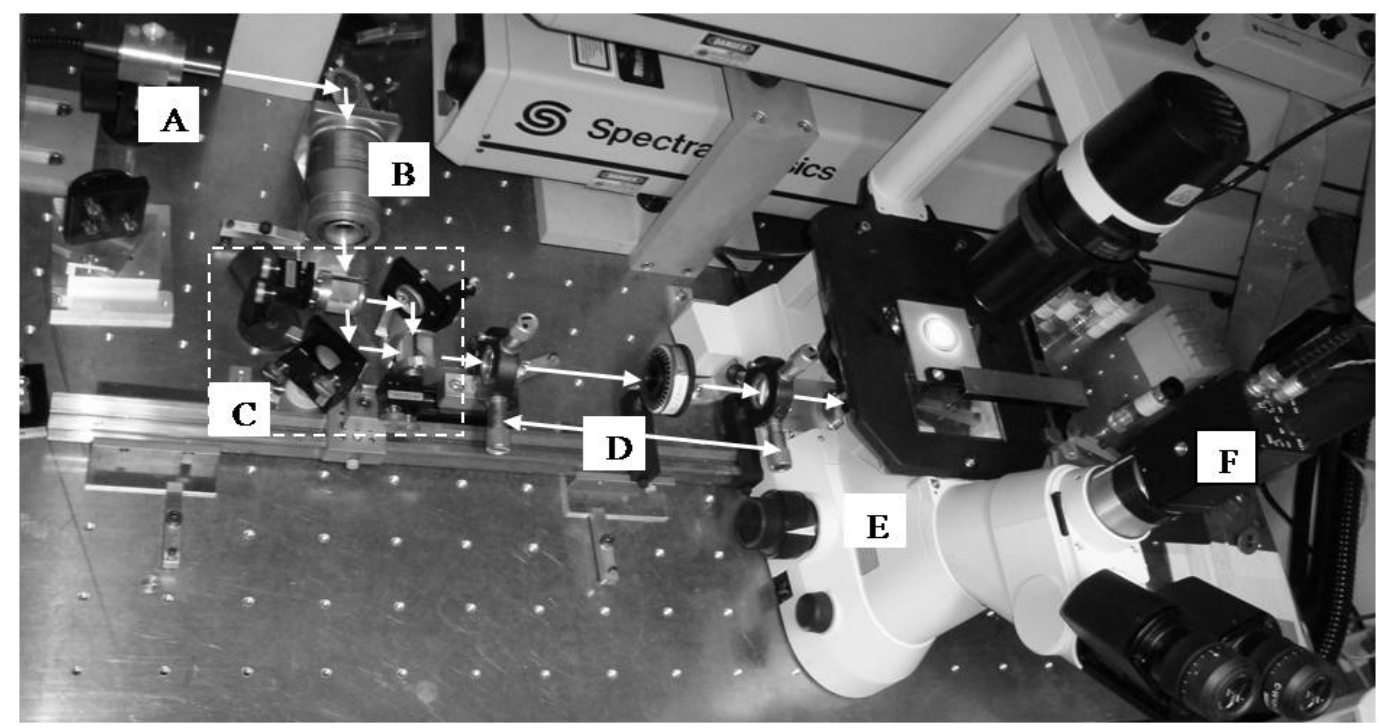

Fig.1. Assembly of a double trap system. A) Exit of the infrared laser beam. B) Polarization rotor. C) Beam splitter system. D) Telescopic system. E) Inverted microscope. F) CCD Camera.

A simplified schematic to show how the two traps are generated by division of beam polarization and manipulated by means of the mechanical controls of the mirrors $M_{1}$ and $M_{2}$ is shown in figure 2. The telescopic system has been omitted for simplicity.

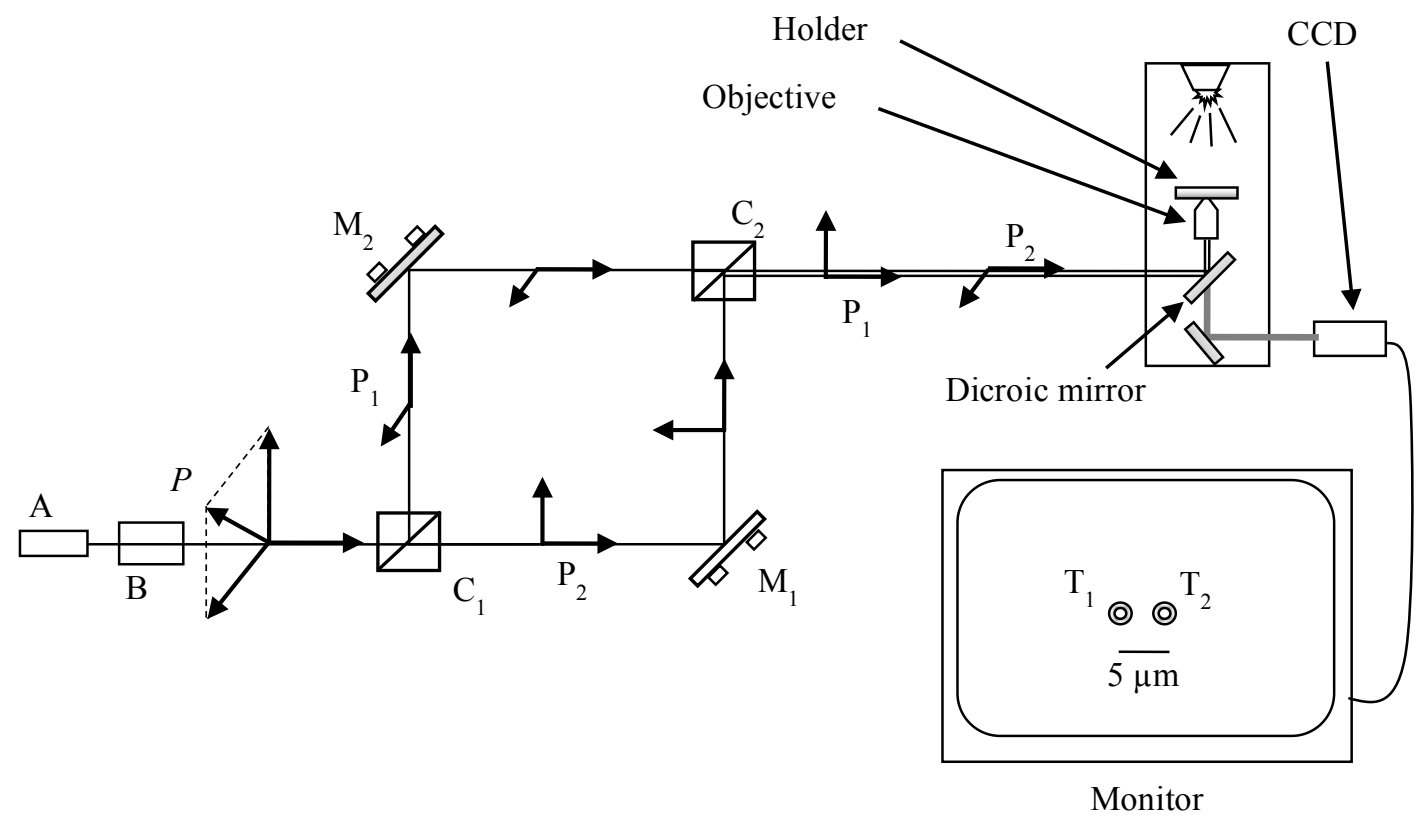

Fig.2. Simplified schematic of generation of two trap points $\mathrm{T}_{1}$ and $\mathrm{T}_{2}$ at the holder plane.

The ray coming from the IR laser A is linearly polarized and is configured horizontally. The polarization rotor $\mathrm{B}$ is used later so that the ray impacts with a linear polarization at approximately $45^{\circ}$ with respect to the horizontal axis on the beam splitter $C_{1}$ and that is the reason why these double optical tweezers can be 
considered as composed of two rays with orthogonal polarization components horizontally and vertically (respectively $P_{2}$ y $P_{1}$ ) and with equal power. When the ray leaves the first beam splitter, the transmitted component that goes to mirror $\mathrm{M}_{1}$ continues with its horizontal polarization state, while the reflected component that goes to mirror $\mathrm{M}_{2}$ has a vertical polarization. These two rays labeled as $\mathrm{P}_{1}$ and $\mathrm{P}_{2}$ in figure 2 are collected by beam splitter $\mathrm{C}_{2}$ but continue traveling as independent rays, making possible the two trap points into the holder of the microscope, which are visualized in the monitor labeled as $\mathrm{T}_{1}$ and $\mathrm{T}_{2}$. The actual distance between the trap points is $5 \mu \mathrm{m}$, as can be seen in figure 2. This approach is based on comparisons made with some crystals with dimensions previously established [7]. In each one of the mirrors $M_{1}$ and $M_{2}$, two mechanical controls allow the movement of the traps in the horizontal plane in holder, since the variations of depth are given by the microscope mechanical focus system.

\section{Experimental Results}

In all the investigated cases that we will show in this section, the capture power is proportional to the minimum force required to obtain a stable capture. The laser used in these experiences has a tunable power, which permits us to calibrate the trap easily, in order to get the necessary force for a strong capture, which can be achieved with any power that is slightly higher over the minimal value required for the capture. The beam power has been measured at the microscopy holder, from which we found that in each trap only the $5 \%$ of the initial power of the laser was measured. It means that the power of the two traps in the holder, is approximately $10 \%$ of the tuned power of the laser. These losses are due to the passing of the beam through the optical elements present in the experimental setup.

\section{3.a. Capture and control of microorganisms}

The double optical tweezers were used to capture and manipulate an elongated bacilliform bacterium with $12.0 \mu \mathrm{m}$ in size approximately, taking it by its two ends. The $12.0 \mu \mathrm{m}$ long bacterium was present in the samples of crystals, but it was not possible to determine its exact name, although this exact name is not important for our purposes. The capture and manipulation of microorganisms is more difficult than the manipulation of crystals because those offer resistance to capture, writhing and agitating to get liberated from the traps. In this case a $150 \mathrm{~mW}$ power laser was used, and due to several losses, each trap registered an approximate final power of $7.5 \mathrm{~mW}$. The controlled rotation of bacterium is appreciated in figure 3 , where a succession of twelve pictures extracted from experimental video is shown. The time elapsed between each one of the images was five seconds. With this capture method it was possible to fix one of the two ends and to rotate the bacterium moving the other end by means of the controls of the mirror associated with the second trap. This procedure is generally carried out step by step, executing small vertical and horizontal displacements of the order of $0.5 \mu \mathrm{m}$. It is also possible to transfer or to produce other movements to the microorganism manipulating both traps simultaneously, but this would demand a more sophisticated electromechanical control of mirrors. The bacterium is in a watery medium whose refraction index is 1.33 .

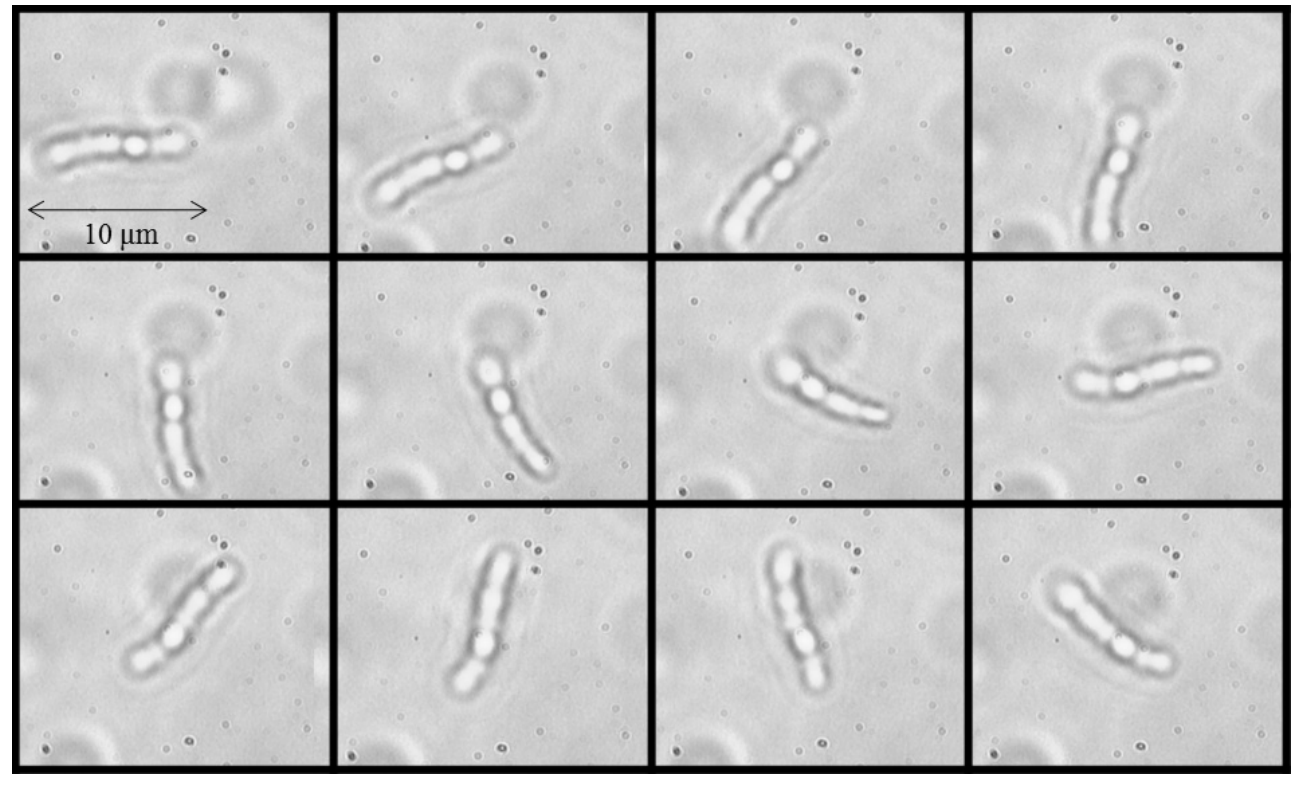

Fig. 3. Video-pictures sequence that shows the controlled rotation of a microscopic bacterium. 


\section{3.b. Control of irregular microscopic crystals}

For this capture an exit power of $200 \mathrm{~mW}$ was used, with which the final power achieved was $10 \mathrm{~mW}$ in each trap. In the case of irregular glasses many rotations and deviations of the ideal behavior of capture were presented due to their irregularities and different curvatures, however, trapping situations were obtained that allowed the manipulation of these crystals in two dimensions. In figure 4, a complete rotation of an irregular crystal is shown, which was carried out following a procedure similar to that of the previous case. This capture was more unstable than in the case of the bacterium because the irregular surface of the glass produces variations in the exerted force. The time elapsed between two series pictures is four seconds.

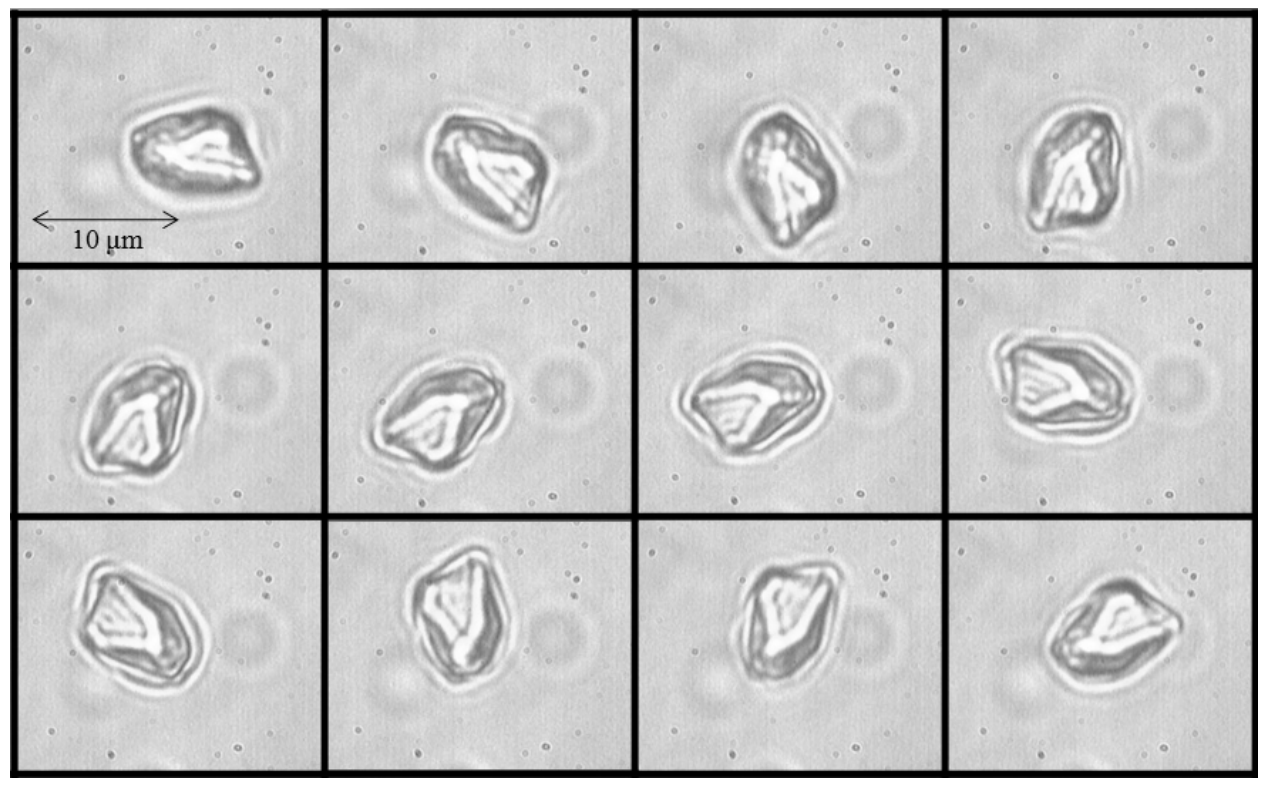

Fig. 4. Video-pictures sequence that shows the controlled rotation of an irregular-shaped crystal.

\section{3.c. Control of regular shaped crystals}

Figure 5 illustrates a sequence of twelve pictures achieved during the manipulation in two dimensions of a microcrystal NBD-C8 (4-n-Octylamino-7-Nitrobenz-2-Oxa-1,3-Diazole, kindly provided by Dr. S. FeryForgues from Toulouse University, France) of approximate dimensions $10.0 \mu m \times 4.0 \mu m \times 0.5 \mu m$. The capture was achieved using a $100 \mathrm{~mW}$ exit power laser, this means that in each trap the power was $5 \mathrm{~mW}$. The crystal was caught and manipulated parallel to its smaller face.

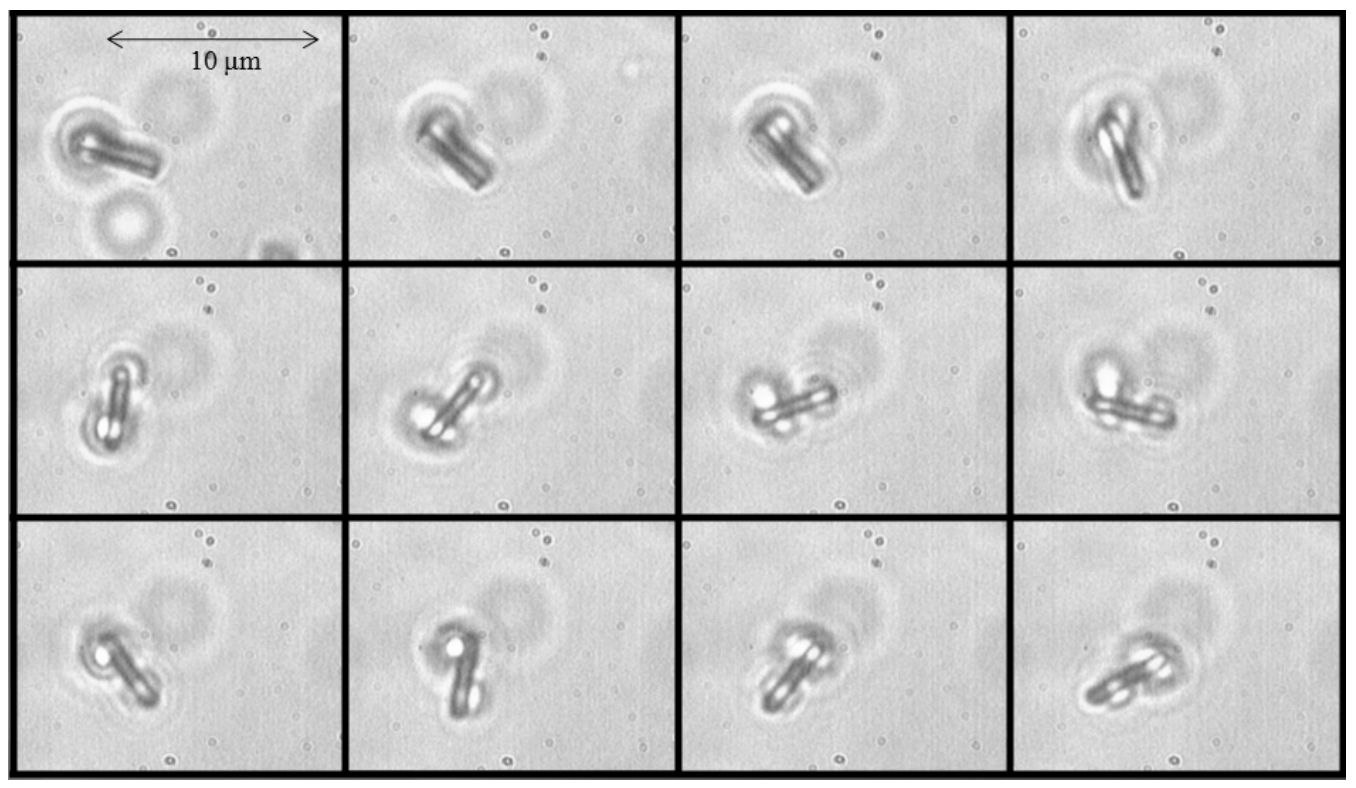

Fig. 5. Video-pictures sequence that shows the controlled rotation of a $10.0 \mu \mathrm{mx} 4.0 \mu \mathrm{mx} 0.5 \mu \mathrm{m}$ parallelepiped-shaped crystal. 
Figure 5 shows the smallest rectangular profile in the parallelepiped of $4.0 \mu m \times 0.5 \mu m$. There was a lapse of eight seconds between each couple of pictures. The capture was quite unstable and it was the most difficult one to achieve because in this case the capture points don't work in the same way as in the cited published papers [6], but rather they act as points where the forces are focused, thanks to the objective of the microscope.

In this case the capture positions are inside the parallelepiped and the rotations had to be much finer to avoid the loss of the capture. In this setup it was not possible to produce any movement of the glass in perpendicular direction to the observation plane. To exert control of the depth dimension another assembly is required.

\section{Conclusions and perspectives}

Regular and irregular microcrystals were captured and manipulated in two dimensions as well as an elongated bacterium, executing complete rotations and displacements using a stabilized IR laser of tunable power in a low cost double optic tweezers manipulated mechanically by means of mirrors. The rotational dynamics of a micrometric parallelepiped in two dimensions was controlled, parallel to one of their faces. Although the mechanical control of the tweezers allows a limited action range as for displacements, it can be said that the capture is sufficiently stable so that the tweezers can be used in other applications such as biological investigations or study and characterization of materials or microstructures. The next stage is to build an electronic movement controller for the mirrors. These can be controlled by four step-motors from an electronic card with a build on microcontroller, or, using another programmable device. It is also possible to generate multiple traps and to control them from a computer by using spatial light modulators SLM; but this alternative is very expensive in our environment. For this reason, the experimental setup of our work becomes important due to its low cost.

The multiple applications of the optical tweezers are important to develop a more precise dynamic control of micro-crystals and microorganisms. In this process the next step is the three-dimensional control of the same ones, for that to be achieved is necessary to implement a holographic optical trap that requires a spatial light modulator (SLM). It is hoped to develop the technique of three-dimensional holographic capture of regular and irregular bodies, which can be used in many areas such as: the production process of micro and nano-machines; the manipulation of molecules and microorganisms in biology, medicine and genetics, and in the controlled growth and complete manipulation of micro and nano-glasses as well as micro and nano-structures [8].

\section{Acknowledgements}

This work was possible thanks to collaboration with the Aimé Cotton Laboratory, at Paris Sud 11 University. We want to thank to director Prof. Pierre Pillet. Javier V V wishes to thank the Instituto Tecnológico Metropolitano de Medellín, ITM for the economic support. Thanks also to: Alex Smith, Liliam Velásquez and Mauricio Echeverry for their collaboration. 